\title{
Report from the AMRSN-Antimicrobial Resistance Surveillance Network; data from 2013 to 2017 spanning four geographically distant tertiary care hospitals in India
}

SM Rudramurthy ${ }^{1}$, M Dhaliwal ${ }^{1}$, Singh R ${ }^{2}$, I Xess ${ }^{3}$, SJ Michael ${ }^{4}$, H Kaur $^{1}$, A Ghosh ${ }^{1}$, Walia K ${ }^{5}$, V Ohri ${ }^{5}$, A Chakrabarti ${ }^{1}$

${ }^{1}$ Postgraduate Institute of Medical Education and Research, Chandigarh, India; 2 Jawaharlal Institute of Postgraduate Medical Education and Research, Puducherry, India; ${ }^{3}$ All India Institute of Medical Sciences, New Delhi, India; ${ }^{4}$ Christian Medical College, Vellore, India; ${ }^{5}$ ndian Council of Medical Research, New Delhi, India

\section{Objectives:}

Establish "Antifungal Resistance" in India.

Monitor trends in fungal species distribution, susceptibility landscapes, creation of a national database and eventually establish guidelines on combating the spread of antifungal resistance in India.

Methods: This multicentre study was initiated in 2013 and is ongoing. Four tertiary care centres are collaborating centres.

Clinical details and the yeasts isolated were collected.

All the yeasts were identified by MALDI-TOF and some by DNA Sequencing.

Antifungal susceptibility testing was performed as per broth microdilution technique of CLSI M27 protocol.

Interpretation of the breakpoints were done as per the recent guidelines.
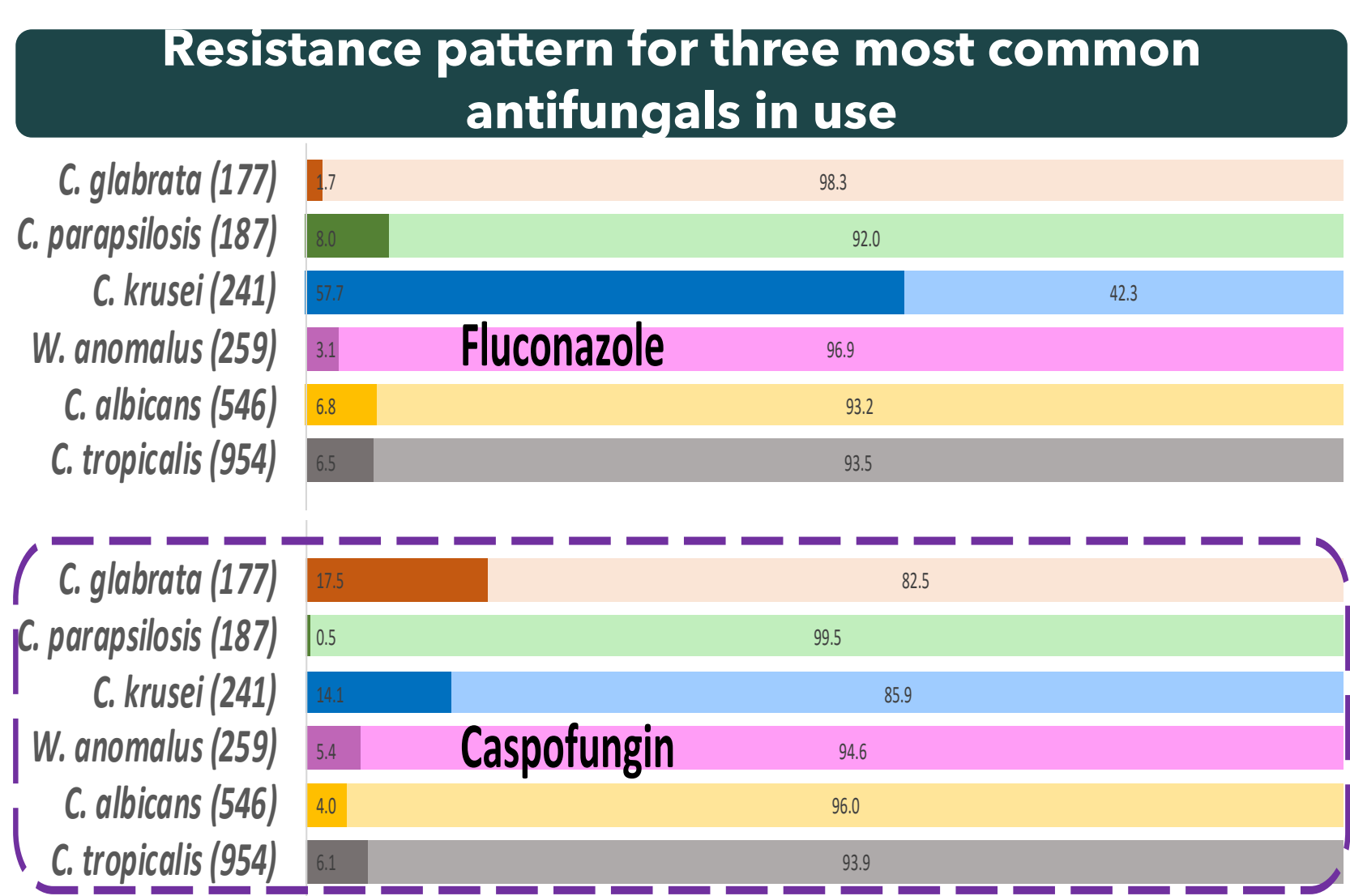

C. glabrata (177) C. parapsilosis (187)

C. krusei (241)

W. anomalus (259) $0.0 \quad$ Amphotericin-B

C. albicans (546) 18

C. tropicalis (954)

Conclusion: The spectrum of Candida species causing invasive infections has expanded. $W$. anomalous is emerging an agent of invasive candidiasis and is seen as third most common yeast. Fluconazole and caspofungin resistance to C. tropicalis, is of concern. Fluconazole resistance with C. albicans is as per earlier rates but increasing trend of resistance to voriconazole and caspofungin has been noted. C glabrata had low fluconazole resistance.
Resistance patterns among various yeast species isolated during the network study

\begin{tabular}{|cccccc|}
\hline & \multicolumn{2}{c}{ MIC/MEC } & \multicolumn{2}{c|}{$\%$ by category } \\
Species Antifungal Tested & Range & $50 \%$ & $90 \%$ & S & R \\
(Tot 2978) & & & & &
\end{tabular}

C. tropicalis Amphotericin-B

(954) Caspofungin

Anidulafungin

Micafungin

Fluconazole

Voriconazole

Posaconazole

Itraconazole

$0.03-8$

0.5

$0.03-16$

$0.02-4$

0.12

$0.02-16$

0.03-64

0.03-4

0.03-16

$0.03-8$

0.03

0.12

0.5

0.06

0.12

0.5

0.03-2

0.5

C. albicans Amphotericin-B

0.03-64

(546) Caspofungin

Anidulafungin

Micafungin

Fluconazole

Voriconazole

Posaconazole

Itraconazole

0.02-4

$0.02-2$

0.03-64

$0.03-16$

$0.03-8$

0.03-16

0.12

0.03

0.12

0.25

0.03

0.12

0.12

1
0.5

97.6

2.4

0.12

0.25

4

0.25

0.25

0.12

93.9

98.8

98.4

93.5

97.2

99.4

98.4

1

98.2

96.0

0.12

0.12

2

0.5

0.5

0.5

98.7

98.7

93.2

93.4

99.1

95.8

6.1

1.2

1.6

6.5

2.8

0.6

1.6

W. Amphotericin-B

0.03-1

0.5

anomalus Caspofungin

$0.03-4$

0.25

0.03-2

0.03-2

Micafungin

Fluconazole

Voriconazole

Posaconazole

Itraconazole

$0.12-64$

0.03-1

0.03-16

0.03-1

0.03

0.12

2

0.12

0.25

0.25

1

100.0

1.8

4.0

1.3

1.3

6.8

6.6

0.9

4.2

C. krusei Amphotericin-B

0.03-4

(241) Caspofungin

Anidulafungin

Micafungin

Fluconazole

Voriconazole

Posaconazole

Itraconazole

0.03-8

0.03-4

0.03-4

$0.12-64$

0.03-8

0.03-0.5

0.03-1

0.12

0.25

4
0.5

1
0.5

94.6

97.7

97.3

96.9

99.6

98.8

97.7

$\mathrm{Nil}$

5.4

2.3

2.7

3.1

0.4

1.2

2.3

82.6

7.9

0.25

0.12

0.12

8

0.25

0.25

0.25

85.9

14.1

0.5

95.0

5.0

0.5

95.0

42.3

5.0

57.7

0.5

98.3

1.7

$100.0 \mathrm{Nil}$

0.5

97.5

2.5

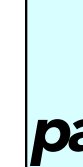

C. Amphotericin-B

0.03-2 $\quad 0.5$

0.03-16

98.4

99.5

1.6

(187) Anidulafungin

Micafungin

Fluconazole

Voriconazole

Posaconazole

Itraconazole

0.03-8

$0.03-4$

$0.12-64$

0.5

0.5

0.5

0.03

$0.03-4$

0.06

0.03-4

0.12

99.5

100.0

92.0

98.9

99.5

0.25

97.9

C. glabrata Amphotericin-B

0.12-8 0.5

(177) Caspofungin

Anidulafungin

0.03-4

0.12

0.02-4

0.06

Micafungin

Fluconazole

0.03-1

Voriconazole

$0.12-64$

Posaconazole

0.03-8

0.12

0.03-8

0.12

Itraconazole

$0.03-2$

0.25

\begin{tabular}{|c|c|}
\hline & 0.12 \\
\hline-4 & 1 \\
\hline 8 & 0.12 \\
\hline 2 & 0.25 \\
\hline
\end{tabular}

\begin{tabular}{|c|c|c|}
\hline 1 & 96.0 & 4.0 \\
\hline 0.5 & 82.5 & 17.5 \\
0.12 & 97.7 & 2.3 \\
0.12 & 91.0 & 9.0 \\
8 & 98.3 & 1.7 \\
1 & 88.7 & 11.3 \\
0.5 & 97.7 & 2.3 \\
0.5 & 94.4 & 5.6 \\
\hline
\end{tabular}

